\title{
Das Schibboleth der Psychoanalyse. LIMINALITÄT BEI FREUD
}

\author{
ACHIM GEISENHANSLÜKE
}

\section{Liminalität transdisziplinär}

Das Thema der Liminalität, das ursprünglich vor allem mit den Namen von Ethnologen und Anthropologen wie Arnold van Gennep und Victor Turner verbunden war, ${ }^{1}$ hat in den letzten Jahren in den Geisteswissenschaften überraschende Erweiterungsmöglichkeiten erfahren. So schreibt etwa die Soziologin Eva Illouz in ihrer vielbeachteten Studie Der Konsum der Romantik einleitend, »dass sich die utopische Dimension romantischer Liebe aus einer spezifischen Kategorie des Religiösen herleitet, die der Anthropologe Victor Turner als >Liminalität` oder Schwellenzustand bezeichnet hat . $^{2}$ Der Rekurs auf Turner, den Illouz in ihrer Studie unternimmt, ist ein Beispiel unter vielen, das von der Aktualität des ethnologischen Liminalitätsbegriffs in anderen Disziplinen zeugt. Nicht nur in der Soziologie richtet sich die Aufmerksamkeit verstärkt auf Schwellenrituale. Der Zusammenhang von Ritual und Performativität hat, wie zum Beispiel die Arbeiten von Erika Fischer-Lichte und Wolfgang Braungart zeigen, ${ }^{3}$ sowohl die Theater- als auch die Literaturwissenschaft der letzten Jahre beschäftigt. In den Literaturwissenschaften gibt der Leitbegriff der Schwelle unterschiedlichsten Forschungsrichtungen Raum. So unternimmt Nicholaus Saul unter dem Titel Schwellen. Germanistische Erkundungen einer Metapher den Versuch, den bisher von Lothar Pikulik und anderen vor allem auf die Romantik bezogenen Begriff der Schwelle als Metapher und Denkfigur geltend zu machen, um

1 Vgl. Arnold van Gennep: Übergangsriten (Les rites de passage), Frankfurt a.M., New York 1999; Victor Turner: Das Ritual. Struktur und AntiStruktur, Frankfurt a.M., New York 2005.

2 Eva Illouz: Der Konsum der Romantik. Liebe und die kulturellen Widersprüche des Kapitalismus, Frankfurt a.M., New York 2003, S. 12f.

3 Vgl. Erika Fischer-Lichte/Wolfgang Braungart: Ritual und Literatur, Tübingen 1996. 
»den jeweiligen Erkenntnisgegenständen eingeschriebenen Zweideutigkeiten nachzugehen «. ${ }^{4}$ Im Blick auf Phänomene der »Limitation und Transgression in Literatur und Ästhetik« haben Claudia Benthien und Irmela Marei Krüger-Fürhoff dagegen Michel Foucaults Begriff der Überschreitung in Anspruch genommen, um eine Kultur des Zwischen zu etablieren, die in Dirk Hohnsträters Lob des Grenzgängers mündet, demzufolge »Grenzen nicht als Linien zwischen zwei Seiten, sondern als Streifen, als Zwischenräume gedacht werden $\aleph^{5}$ müssen.

Angesichts der Vielfalt der unterschiedlichen Beiträge zum Thema der Liminalität stellt sich zugleich die Frage nach der theoretischen Grundlegung des Begriffes im Zwischenraum von Ethnologie und Diskursanalyse. Der vorliegende Beitrag knüpft an mögliche Erweiterungen des Liminalitätsbegriffes in anderen Disziplinen an, möchte im Blick auf den Zusammenhang von Liminalität und Literalität, der im Mittelpunkt der Tagung steht, jedoch zugleich den Akzent verschieben, indem er Freuds Psychoanalyse zum Gegenstand der Diskussion nimmt. Ausgangspunkt der folgenden Überlegungen ist nicht nur die Tatsache, dass sich im Denken Freuds auch in seinen unterschiedlichen Phasen immer wieder die Auseinandersetzung mit liminalen Phänomenen beobachten lässt, sondern zugleich der eigentümliche Ort von Freuds eigenem Schreiben im Zwischenraum von Wissenschaft und Fiktion. Das gilt für die frühe, gemeinsam mit Fließ entwickelte Theorie von der grundsätzlichen Bisexualität des menschlichen Wesens wie für die Auseinandersetzung mit dem Phänomen des Traums als einem Übergangsstadium zwischen Schlaf und Erwachen. Eine besondere Bedeutung gewinnen Theorie und Schreiben der Liminalität bei Freud in seinen späten Ausführungen zur Ich-Theorie, in denen die frühe Unterscheidung von Bewusstem und Unbewusstem eine Revision erfährt, die zugleich zu der Begründung eines Oberflächenmodells führt, in dem das Phänomen der Liminalität neu fundiert wird. Die folgenden Ausführungen konzentrieren sich nach einem kurzen Seitenblick auf die Bedeutung der Liminalität für das Denken Walter Benjamins daher auf zwei zentrale Schriften Freuds, Die Traumdeutung und Das Ich und das Es.

4 Nicholas Saul/Daniel Steuer/Frank Möbius/Birgit Illner: Schwellen. Germanistische Erkundungen einer Metapher, Würzburg 1999, S. 9. Zur Romantik vgl. Lothar Pikulik: »Schwelle und Übergang. $\mathrm{Zu}$ einem Schlüsselmotiv der Romantik«, in: Aurora 53 (1993), S. 13-24, hier S. 13-14.

5 Dirk Hohnsträter: »Im Zwischenraum. Ein Lob des Grenzgängers«, in: Claudia Benthien/Irmela Marei Krüger-Fürhoff (Hg.): Über Grenzen. Limitation und Transgression in Literatur und Ästhetik, Stuttgart, Weimar 1999, S. 231-244, hier S. 244. 


\section{Schwellenkunde: Walter Benjamin}

Einer der ersten Theoretiker, der den Begriff der Schwelle zum Gegenstand seiner Untersuchungen genommen hat, ist Walter Benjamin. Wie Winfried Menninghaus in seiner Arbeit Schwellenkunde gezeigt hat, ist der Ausgangspunkt von Benjamins Überlegungen zur Schwelle nicht nur, wie bereits der Titel es nahelegt, das Passagen-Werk, »auch die >prominentesten< Literaturinterpretationen Benjamins - der Wahlverwandtschaften-Aufsatz, die Tragödientheorie des Trauerspielbuchs, das Proust-, Kraus- und vor allem das Kafka-Portrait - sind wesentlich auf die Erkenntnis von Schwellen zentriert «. ${ }^{6}$

Den Begriff der Schwellenkunde kann Menninghaus für Benjamins Passage des Mythos geltend machen, da Benjamin einer der Ersten ist, der scharf zwischen Schwelle und Grenze unterscheidet: »Die Schwelle ist ganz scharf von der Grenze zu scheiden. Schwelle ist eine Zone. Wandel, Übergang, Fluten liegen im Worte >schwellen` und diese Bedeutung hat die Etymologie nicht zu übersehen. Andererseits ist es notwendig, den unmittelbaren tektonischen und zeremonialen Zusammenhang festzustellen, der das Wort zu seiner Bedeutung gebracht. « ${ }^{7}$ Wie das Passagen-Werk zeigt, stellt das Denken Walter Benjamins einen der prominentesten Versuche dar, den Begriff der Schwelle in das Zentrum der historischen Erkenntnis zu stellen. Dabei bindet Benjamin den Begriff der Schwelle in ähnlicher Weise wie die Ethnologie an archaische Formen zurück, die in der Moderne verstummt zu sein scheinen. Unter dem Stichwort »Rites de passage« notiert Benjamin im Passagen-Werk: »Wir sind sehr arm an Schwellenerfahrungen geworden. Das Einschlafen ist vielleicht die einzige, die uns geblieben ist. ${ }^{8}$ Scheint die Schwellenerfahrung in der Moderne nur noch eine marginale Rolle einzunehmen, so wird die Literatur zu dem Ort, an dem liminale Phänomene weiter bestehen können. Wenn Benjamin den Begriff der Schwelle in den Kontext von Schlaf, Traum und Erwachen stellt, dann spielt er auf der einen Seite auf die Vertreter der modernen Literatur an, die er für repräsentativ hält, auf Kafka und Proust. Auf der anderen Seite setzt er sich kritisch mit der Psychoanalyse auseinander, die den Traum ebenfalls als ein Schwellenphänomen zur Geltung gebracht hat. Trotz seiner, wie Susan Buck-Morss

6 Winfried Menninghaus: Schwellenkunde. Walter Benjamins Passage des Mythos, Frankfurt a.M. 1986, S. 8.

7 Walter Benjamin: Das Passagen-Werk. Erster Band, Frankfurt a.M. 1982, S. 618 .

8 Ebd., S. 617. 
gezeigt hat, ${ }^{9}$ weitgehend kritischen Rezeption der Psychoanalyse ergeben sich daher zugleich Gemeinsamkeiten zwischen Benjamin und Freud, die nicht nur den Gegenstand, sondern auch die Form der Auseinandersetzung betreffen. Über Benjamins Schwellenkunde notiert Menninghaus: »Passage ist sein Werk also in einem dreifachen Sinn: in seiner geschichtsphilosophischen Intention, seiner wissenschaftlichen Form und seinem Hauptgegenstand. ${ }^{10}$ Wie im Folgenden zu zeigen sein wird, gilt Ähnliches auch für Freud.

\section{Die Schwelle des Traums}

Dass sich die Grenzen zwischen Wissenschaft und Literatur in Freuds Denken unaufhörlich verschieben, zeigt bereits der Blick auf seine frühen Schriften. So schreibt Freud im Anschluss an seine Falldarstellungen in den Schriften über Hysterie: »[...] es berührt mich selbst noch eigentümlich, daß die Krankengeschichten, die ich schreibe, wie Novellen zu lesen sind, und daß sie sozusagen des ernsten Gepräges der Wissenschaftlichkeit entbehren. ${ }^{11}$ Was als koketter Einwand gegen die Wissenschaftlichkeit des eigenen Unterfangens gelesen werden kann, ist zugleich ein erstes Zeichen für die Symbiose, die Literatur und Wissenschaft in Freuds Denken eingehen. Nähern sich die Studien über Hysterie der Gattungsform der Novelle, so lässt sich die Traumdeutung, wie von der Forschung häufig hervorgehoben worden ist, zugleich als Autobiographie lesen. Das hat Freud selbst nahegelegt, wenn er in der Einleitung zur zweiten Auflage bemerkt: »Für mich hat dieses Buch nämlich noch eine andere subjektive Bedeutung, die ich erst nach seiner Beendigung verstehen konnte. Es erwies sich mir als ein Stück meiner Selbstanalyse, als meine Reaktion auf den Tod meines Vaters, also auf das bedeutsamste Ereignis, den einschneidendsten Verlust im Leben eines Mannes. « (GS II, 1) Die Selbstanalyse Freuds verbindet sich nicht zuletzt vor dem Hintergrund des Ödipuskomplexes in der Traumdeutung mit der Auseinandersetzung mit dem toten Vater. Ilse Grubrich-Simitis hat die Traumdeu-

9 Vgl. Susan Buck-Morss: Dialektik des Sehens. Walter Benjamin und das Passagen-Werk, Frankfurt a.M. 1993, S. 556f.

10 W. Menninghaus: Schwellenkunde, S. 49f.

11 Sigmund Freud: Gesammelte Werke. Chronologisch geordnet. Unter Mitwirkung von Marie Bonaparte, Prinzessin Georg von Griechenland, herausgegeben von Anna Freud, Frankfurt a.M. 1999, Bd. 1, S. 227. Im Folgenden im Text abgekürzt: lateinische Ziffer für den Band, arabische Ziffer für die Seitenangabe. 
tung daher als eine »mosaikartige Tiefen-Autobiographie $\aleph^{12}$, als $\gg$ sein persönlichstes, als quasi-autobiographisches Buch« bezeichnet. Als Reise zum toten Vater gewinnt die Traumdeutung zugleich die Qualitäten einer literarischen katábasis, einer Hadesfahrt, die Freud in Jenseits des Lustprinzips weiterführt. ${ }^{13}$ Schon in dieser Verquickung von literarischen und wissenschaftlichen Momenten im Werk Freuds wird deutlich, wie sehr die Psychoanalyse von Schwellen und Übergängen geprägt ist.

Das gilt in besonderem Maße für Freuds »Jahrhundertbuch«, Die Traumdeutung, auf dessen Erscheinungsjahr Freud besonders stolz zu sein schien: »Die Psychoanalyse ist sozusagen mit dem zwanzigsten Jahrhundert geboren; die Veröffentlichung, mit welcher sie als etwas Neues vor die Welt tritt, meine >Traumdeutung`, trägt die Jahreszahl 1900.« (GS XIII, 405) Nicht nur steht das Erscheinen der Traumdeutung zwischen 1899 und 1900 auf der Schwelle der beiden Jahrhunderte. Freud zufolge markiert die Traumdeutung in ihrer Bedeutung als Schibboleth selbst so etwas wie eine Schwelle, die den Zugang zur Psychoanalyse eröffnet oder auch verhindert. So schreibt Freud in den Neuen Vorlesungen zur Einführung in die Psychoanalyse: »Die Traumlehre ist seither auch das Kennzeichnendste und Eigentümlichste der jungen Wissenschaft geblieben, etwas, wozu es kein Gegenstück in unserem sonstigen Wissen gibt«, ein »Schibboleth«. Mit der Kennzeichnung der Traumdeutung als Schibboleth will Freud noch einmal die Einzigartigkeit seines Jahrhundertbuches unterstreichen und zugleich auf die verborgenen jüdischen Quellen der Psychoanalyse eingehen: »Es ist vielleicht auch kein bloßer Zufall, daß der erste Vertreter der Psychoanalyse ein Jude war« (GS XIV, 110), heißt es in der Schrift Die Widerstände gegen die Psychoanalyse. Als Schibboleth will Freud die Traumdeutung verstanden wissen, da sie über die Zuordnung zu den Anhängern oder den Gegnern der Psychoanalyse entscheidet. In ähnlicher Weise, wie Jacques Derrida die Dichtung Paul Celans mit dem hebräischen Begriff des Schibboleths unter das Zeichen von Beschneidung, Datierung und Chiffrierung gestellt hat, ${ }^{14}$ steht die Traumdeutung an der Schwelle des Jahrhunderts als Passwort, das an den Fluten des Jordans über Leben und Tod entscheidet.

Dass es in der Traumdeutung um die Frage von Leben und Tod geht, hatte schon Freuds Bemerkung angedeutet, sein Jahrhundertbuch sei zugleich die Reaktion auf den Tod des Vaters. Die Bedeutung der Traum-

12 Jean Starobinski/Ilse Grubrich-Simitis/Mark Solms: Hundert Jahre >Traumdeutung< von Sigmund Freud. Drei Essays, Frankfurt a.M. 2000, S. 7 und S. 67.

13 Vgl. Isabel Platthaus: Höllenfahrten. Die epische katábasis und die Unterwelten der Moderne, München 2004.

14 Vgl. Jacques Derrida: Schibboleth. Für Paul Celan, Wien 1986. 
deutung als einer symbolischen Hadesfahrt, die Freud unternimmt, indem er sich in das Reich des Schlafes und des Traums hinab begibt, flankiert noch das Motto des Buches: »Flectere si nequeo superos, acheronta movebo« (Weigern's die droben, so werde ich des Abgrunds Kräfte bewegen). Der untergründigen Bedeutung des Mottos für die gesamte Traumdeutung ist Jean Starobinski nachgegangen. »Dieser Vers Vergils stellt, wie wir wissen und noch genauer sehen werden, in einer mächtigen Allegorie den Umweg dar, den die Kraft des Begehrens einschlägt, wenn der >gerade Weg८ ihr >versperrt « ist $«,{ }^{15}$ hält Starobinski fest, um die Bewegung der Psychoanalyse als Abstieg in eine Tiefe zu fassen, die durch das Vergilzitat zugleich als eine moderne Form der katábasis erscheint. Wie Starobinski deutlich macht, verbindet sich das Motiv der Hadesfahrt bei Freud mit dem Bild wissenschaftlichen Fortschritts, das Freud einleitend bemüht, wenn er den Weg der Psychoanalyse zugleich als einen Aufstieg zu einer Anhöhe angibt:

Wenn man einen engen Hohlweg passiert hat und plötzlich auf einer Anhöhe angelangt ist, von welcher aus die Wege sich teilen und die reichste Aussicht nach verschiedenen Richtungen sich öffnet, darf man einen Moment lang verweilen und überlegen, wohin man sich zunächst wenden soll. Ähnlich ergeht es uns, nachdem wir diese erste Traumdeutung überwunden haben. Wir stehen in der Klarheit einer plötzlichen Erkenntnis. Der Traum ist nicht vergleichbar dem unregelmäßigen Ertönen eines musikalischen Instruments, das anstatt von der Hand des Spielers, von dem Stoß einer äußerlichen Gewalt getroffen wird, er ist nicht sinnlos, nicht absurd, setzt nicht voraus, daß ein Teil unseres Vorstellungsschatzes schläft, während ein anderer zu erwachen beginnt. Er ist ein vollgültiges psychisches Phänomen, und zwar eine Wunscherfüllung. (GS II, 127)

Starobinski, der Freuds Darstellung mit Descartes' Erkenntnisanspruch vergleicht, hebt in diesem Zusammenhang hervor: »Das Bild ist stets das eines fortschreitenden Ganges zu neuen Entdeckungen, zu Horizonten, die sich auf immer umfassendere Unternehmungen öffnen. ${ }^{16}$ Hinter dem optimistischen Bild des wissenschaftlichen Fortschritts und der damit verbundenen »Klarheit einer plötzlichen Erkenntnis« öffnet sich aber zugleich eine weitaus düsterere Darstellung, die das einleitend zitierte Bild des Hohlwegs als Metapher für Freuds Lebenskrise, Didier Anzieu zufolge für eine $»$ Krise der Lebensmitte $~^{17}$, deuten kann. Mit dem Bild des Hohlwegs, der Anhöhe und den unterschiedlichen, sich verzweigenden

15 J. Starobinski: Hundert Jahre >Traumdeutung`, S. 14.

16 Ebd., S. 12.

17 Didier Anzieu: Freuds Selbstanalyse und die Entdeckung der Psychoanalyse, Stuttgart 1990, S. 4. 
Wegen, die sich dem Betrachter eröffnen, verweist Freud auf die unterschiedliche Bedeutung der Schwelle als Übergang von wissenschaftlicher Erkenntnis und autobiographischer Reflexion des eigenen Lebensweges im Kontext einer Krisenerfahrung, die neben Vergils Aeneis zugleich auf Dantes Göttliche Komödie verweist: »Nel mezzo del cammin di nostra vita, Mi ritrovai per una selva oscura." So erscheint die Traumdeutung im Dickicht der Lebensmitte als der verzweifelte Versuch, Orientierung zu gewinnen, indem die Schwelle zur Unterwelt aufgehoben wird und der Abstieg in das dunkle Reich der Träume beginnt. In ähnlicher Weise wie Dante zu Beginn seiner Göttlichen Komödie sucht Freud in der Traumdeutung mit dem Motto aus der Aeneis die symbolische Begleitung Vergils, um seinen Abstieg in die Unterwelt zu beginnen, einen Abstieg, der zugleich auf die Höhe neuester wissenschaftlicher Erkenntnis führen soll. Der Gegenstand seiner Untersuchung, der Traum, erscheint in diesem Zusammenhang selbst als ein Schwellenphänomen, als ein transitorischer Ort zwischen den beiden Polen des Einschlafens und des Erwachens und als symbolischer Stellvertreter für die Schattenwelt der Toten, deren bedrohlicher Macht Freud zu begegnen sucht, indem er den Traum als eine Wunscherfüllung ausgibt. Als Jahrhundertbuch und als Schibboleth markiert die Traumdeutung eine Schwelle, die ihr gegenständliches Korrelat in dem Begriff des Unbewussten gefunden hat, das als Raum, in dem die Gesetze von Kausalität, Raum und Zeit nicht länger gelten, selbst eine Instanz markiert, in der sich die Grenzen der Logik auflösen und die Unterscheidung zwischen Wachen und Träumen allmählich aufgehoben wird. So dient der Begriff des Unbewussten der Psychoanalyse als Prüfstein allen wissenschaftlichen Fortschrittes, der mit der noch jungen Wissenschaft verbunden ist, bis die Auseinandersetzung mit den Folgen des Ersten Weltkrieges eine neue Konzeption des psychischen Apparates notwendig macht.

\section{Das arme Ich}

In der Traumdeutung hatte Sigmund Freud den Begriff des Unbewussten als das eigentliche Korrelat der psychoanalytischen Erkenntnis eingeführt. »Die Traumdeutung aber ist die Via regia zur Kenntnis des Unbewußten im Seelenleben « (GS II, 613), formuliert Freud im Blick auf den Zusammenhang von Traum und Unbewusstem, demzufolge jedem Traum eine Wunscherfüllung zugrunde liege. Die Erfahrungen des Ersten Weltkrieges zwangen Freud dazu, seine frühere Auffassung Schritt für Schritt aufzugeben. An die Stelle des Zusammenhangs von Traum und Wunscherfüllung tritt der von Trauma und Wiederholung, der zu- 
gleich zu einer Revision des frühen Konzepts des Unbewussten führt, die in den metapsychologischen Schriften Jenseits des Lustprinzips und Das Ich und das Es zum Tragen kommt.

Die Kontinuität, die zwischen Jenseits des Lustprinzips und Das Ich und das Es besteht, hat Freud selbst einleitend hervorgehoben. »Nachstehende Erörterungen setzen Gedankengänge fort, die in meiner Schrift >Jenseits des Lustprinzips` begonnen wurden, denen ich persönlich, wie dort erwähnt ist, mit einer gewissen wohlwollenden Neugierde gegenüber stand.« (GS XIII, 237) Freud macht das seit Petrarcas Besteigung des Mont Ventoux zentrale Moment der Neugierde für den Fortschritt wissenschaftlicher Erkenntnis geltend, um seine Ausführungen einzuleiten. Im Unterschied zu Petrarca erreicht Freud die neuen Ergebnisse seiner Forschung jedoch nicht durch den Aufstieg in die Höhen der Bergwelt, sondern durch den Abstieg in die Niederungen der Psyche. Ausgangspunkt seiner Überlegungen ist eine harsche Selbstkritik, die das bisher Erreichte in Frage stellt: »Fünfundzwanzig Jahre intensiver Arbeit haben es mit sich gebracht, daß die nächsten Ziele der psychoanalytischen Technik heute ganz andere sind als zu Anfang« (GS XIII, 16), hält Freud fest, um den Weg der Psychoanalyse von der Frage nach der Deutbarkeit der Träume bis zum Problem der Wiederholung zu kennzeichnen:

Dann aber wurde es immer deutlicher, daß das gesteckte Ziel, die Bewußtmachung des Unbewußten, auch auf diesem Wege nicht voll erreichbar ist. Der Kranke kann von dem in ihm Verdrängten nicht alles erinnern, vielleicht gerade das Wesentliche nicht, und erwirbt so keine Überzeugung von der Richtigkeit der ihm mitgeteilten Konstruktion. Er ist vielmehr genötigt, das Verdrängte als gegenwärtiges Erlebnis zu wiederholen, anstatt es, wie der Arzt es lieber sähe, als ein Stück Vergangenheit zu erinnern. (GS XIII, 16)

Das Gelingen der anamnetischen Kur durch die heilende Kraft der Erinnerung stellt das Wiederholungsprinzip radikal in Frage. So scheinen Freuds Ausführungen einem Eingeständnis des Scheiterns gleichzukommen, das in seinen späten Schriften immer wieder durchscheint.

Vor diesem Hintergrund erfolgt die Korrektur an der frühen Unterscheidung von Bewusstem und Unbewusstem als ein folgerichtiger Schritt, der die Psychoanalyse auf neue Grundlagen stellt, indem sie die neue Terminologie des Es, des Ichs und des Über-Ichs einführt. Erscheint das neue Instanzenmodell zunächst auch als Bruch mit der frühen Konzeption des Unbewussten aus der Traumdeutung, so ergeben sich im Blick auf das Thema der Liminalität jedoch zugleich Kontinuitäten.

Dass es sich in Das Ich und das Es keineswegs um einen radikalen Bruch mit früheren Auffassungen handelt, erläutert Freud einleitend selbst am Begriff des Unbewussten. »Die Unterscheidung des Psychi- 
schen in Bewusstes und Unbewusstes ist die Grundvoraussetzung der Psychoanalyse und gibt ihr allein die Möglichkeit, die ebenso häufigen als wichtigen pathologischen Vorgänge im Seelenleben zu verstehen, der Wissenschaft einzuordnen.« (GS XIII, 239) Als Schibboleth behält der Begriff des Unbewussten seine Funktion, den wissenschaftlichen Anspruch der Psychoanalyse zu legitimieren und von konkurrierenden Modellen abzuheben. Dennoch sieht sich Freud gezwungen, Differenzierungen vorzunehmen, um eine größere begriffliche Klarheit zu erlangen. Freud zufolge existiert das Unbewusste in zweierlei Form: als Vorbewusstes, das grundsätzlich bewusstseinsfähig ist, und als Unbewusstes, das sich dem Bewusstsein durch die Verdrängung entzieht:

Wir heißen das Latente, das nur deskriptiv unbewußt ist, nicht im dynamischen Sinne, vorbewußt; den Namen unbewußt beschränken wir auf das dynamisch unbewußte Verdrängte, so daß wir jetzt drei Termini haben, bewußt (bw), vorbewußt (vbw) und unbewußt (ubw), deren Sinn nicht mehr rein deskriptiv ist. (GS XIII, 241)

Die Unterscheidung zwischen deskriptivem und dynamischem Sinn des Unbewussten führt zur Abgrenzung des Unbewussten vom Vorbewussten. Damit scheint Freud die terminologischen Schwierigkeiten, die sich ihm stellten, zunächst gelöst zu haben. Jenseits terminologischer Fragen ergeben sich für die Psychoanalyse jedoch noch weitere Schwierigkeiten, die in der Instanz des Ichs begründet liegen. »Wir haben uns die Vorstellung von einer zusammenhängenden Organisation der seelischen Vorgänge in einer Person gebildet und heißen diese das Ich derselben. « (GS XIII, 243) In der Traumdeutung hatte das Ich als schlafendes noch keine Rolle gespielt. Das Erwachen der Psychoanalyse aus dem Traum der Wunscherfüllung scheint zunächst mit einer enormen Stärkung der Instanz des Ichs einherzugehen:

An diesem Ich hängt das Bewußtsein, es beherrscht die Zugänge zur Motilität, das ist: zur Abfuhr der Erregungen in die Außenwelt; es ist diejenige seelische Instanz, welche eine Kontrolle über all ihre Partialvorgänge ausübt, welche zur Nachtzeit schlafen geht und dann immer noch die Traumzensur handhabt. Von diesem Ich gehen auch die Verdrängungen aus, welche gewiße seelische Strebungen nicht nur vom Bewußtsein, sondern auch von den anderen Arten der Geltung und Betätigung ausgeschlossen werden sollen. (GS XIII, 243)

Freud führt das Ich als einen Herrscher ein, der sowohl im Wachen wie im Träumen die Kontrolle über die Bewusstseinsfunktionen ausübt. Wenn er das Ich als diejenige Instanz anspricht, von der die Verdrängungen ausgehen, dann spielt er auf einen anderen Sachverhalt an: den $\mathrm{Zu}-$ 
sammenhang von Verdrängung und Widerstand. Als Verantwortlicher für die Verdrängung verkörpert das Ich diejenige Instanz, deren Hauptinteresse im Widerstand gegen die Aufdeckung des Verdrängten besteht. Da der Widerstand für Freud aber selbst als Zeichen des Unbewussten gilt, steht er vor einem neuen Problem. Als Ort der Verdrängung und des Widerstands ist das Ich zugleich von der Macht des Unbewussten getroffen: »Da aber dieser Widerstand sicherlich von seinem Ich ausgeht und diesem angehört, so stehen wir vor einer unvorhergesehenen Situation. Wir haben im Ich selbst etwas gefunden, was auch unbewußt ist, sich gerade so benimmt wie das Verdrängte, das heißt starke Wirkungen äußert, ohne selbst bewußt zu werden, und zu dessen Bewußtmachung es einer besonderen Arbeit bedarf.« (GS XIII, 244) Das scheinbar souveräne Ich, das selbst im Schlaf als Traumzensor noch über den Zugang zum Bewusstsein entscheidet, beherbergt in seinem Herzen diejenige Kraft, die ihm am fremdesten erscheint, das Unbewusste. Die Konsequenz aus Freuds Überlegungen liegt in dem Schluss, dass der Begriff der Verdrängung keineswegs mit dem des Unbewussten zusammenfällt. Vielmehr stellt Freud fest: ^Auch ein Teil des Ichs, ein Gott weiß wie wichtiger Teil des Ichs, kann ubw sein, ist sicherlich ubw.« Damit scheint sich aber die Frage nach einer Form des Unbewussten aufzudrängen, das nicht der Verdrängung entspringt. Die unmittelbare Folge der Annahme eines unbewussten Teils des Ichs ist die Tatsache, »daß der Charakter des Unbewussten für uns an Bedeutung verliert.«(GS XIII, 244f.) Mit dem Unbewussten droht der Psychoanalyse der Verlust ihres Schibboleths, der Verlust ihres Anspruches, eine Wissenschaft zu sein, die sich durch einen ihr eigenen Gegenstand von anderen Formen der Wissenschaft unterscheidet. Vor diesem Hintergrund führt Freud die Begriffe des Es und des Ich ein, um die Psychoanalyse an den eigenen Haaren aus dem Sumpf zu ziehen, in dem sie zu versinken droht.

Den Ausgangspunktspunkt seiner Überlegungen bildet das »Bewußtsein als Oberfläche des seelischen Apparates« (GS XIII, 247). Freud greift auf ein räumliches Modell zurück, um den bewussten Teil des Ichs in ähnlicher Weise wie in Jenseits des Lustprinzips als einen Ort zu lokalisieren, der eine eigentümliche Zwischenposition innehat, derzufolge er in der beständigen Auseinandersetzung mit den inneren Trieben und den äußeren Sinnesreizen zu kämpfen hat. Die Unterscheidung von Ich und Es führt daher zu einer neuen Auffassung von der topischen und dynamischen Struktur des Unbewussten:

Ein Individuum ist nun für uns ein psychisches Es, unerkannt und unbewußt, diesem sitzt das Ich oberflächlich auf, aus dem W-System als Kern entwickelt. Streben wir nach graphischer Darstellung, so werden wir hinzufügen, das Ich umhüllt das Es nicht ganz, sondern nur insoweit das System W dessen Oberflä- 
che bildet, also etwa so wie die Keimscheibe dem Ei aufsitzt. Das Ich ist vom Es nicht scharf getrennt, es fließt nach unten hin mit ihm zusammen. (GS XIII, 251)

Noch einmal unterstreicht Freud die Oberflächenstruktur des Ichs. Damit wird zugleich deutlich, dass es sich bei Freuds Konzept eines auf dem Es nur oberflächlich aufsetzenden Ichs um ein liminales Modell handelt, demzufolge die Grenzen zwischen Es und Ich verschwimmen: Als Oberfläche ist das Ich vom Es ebenso getrennt, wie es mit ihm verbunden ist. Um die eigentümliche Position des Ichs festzuhalten, greift Freud auf ein Gleichnis zurück:

Die funktionelle Wichtigkeit des Ichs kommt darin zum Ausdruck, daß ihm normaler Weise die Herrschaft über die Zugänge zur Motilität eingeräumt ist. Es gleicht so im Verhältnis zum Es dem Reiter, der die überlegene Kraft des Pferdes zügeln soll, mit dem Unterschied, daß der Reiter dies mit eigenen Kräften versucht, das Ich mit geborgten. Dieses Gleichnis trägt ein Stück weiter. Wie dem Reiter, will er sich nicht vom Pferd trennen, oft nichts anderes übrig bleibt, als es dahin zu führen, wohin es gehen will, so pflegt auch das Ich den Willen des Es in Handlung umzusetzen, als ob es der eigene wäre. (GS XIII, 253)

Der Vergleich von Ich und Es mit Reiter und Pferd scheint die zentrale Position des Ichs als Kontrollinstanz zunächst zu bestätigen. Als Herrscher über die Zugänge zum Bewusstsein zwingt der Reiter dem Pferd seinen Willen auf. Das Gleichnis verkehrt die Ausgangsposition jedoch schnell in ihr Gegenteil: In Wirklichkeit, so Freuds Darstellung, gehorcht der Reiter dem Pferd, dessen Willen er als den eigenen auslegt. Die Stellung des Ichs als monarchische Herrscherfigur beginnt zu bröckeln, der König wird zum Bettler:

Aber anderseits sehen wir dasselbe Ich als armes Ding, welches unter dreierlei Dienstbarkeiten steht und demzufolge unter den Drohungen von dreierlei Gefahren leidet, von der Außenwelt her, von der Libido des Es und von der Strenge des Über-Ichs. Dreierlei Arten von Angst entsprechen diesen drei Gefahren, denn Angst ist der Ausdruck eines Rückzuges vor der Gefahr. Als Grenzwesen will das Ich zwischen der Welt und dem Es vermitteln, das Es der Welt gefügig machen und die Welt mittels seiner Muskelaktionen dem Es-Wunsch gerecht machen. Es benimmt sich eigentlich wie der Arzt in einer analytischen Kur, indem es sich selbst mit seiner Rücksichtnahme auf die reale Welt dem Es als Libidoobjekt empfiehlt und dessen Libido auf sich lenken will. Es ist nicht nur der Helfer des Es, auch sein unterwürfiger Knecht, der um die Liebe seines Herrn wirbt. Er sucht, wo möglich, im Einvernehmen mit dem Es zu bleiben, überzieht dessen ubw Gebote mit seinen vbw Rationalisierungen, spiegelt den 
Gehorsam des Es gegen die Mahnungen der Realität vor, auch wo das Es starr und unnachgiebig geblieben ist, vertuscht die Konflikte des Es mit der Realität und wo möglich auch die mit dem Über-Ich. In seiner Mittelstellung zwischen Es und Realität unterliegt es nur zu oft der Versuchung, liebedienerisch, opportunistisch und lügnerisch zu werden, etwa wie ein Staatsmann, der bei guter Einsicht sich doch in der Gunst der öffentlichen Meinung behaupten will. (GS XIII, 286f.)

Eingeführt hatte Freud das Ich als Herrscher über die Zugänge der Motilität, dessen Macht bis in den Schlaf hineinreicht. Am Schluss seiner Abhandlung hat sich die Situation in ihr Gegenteil verkehrt: Das Ich gilt der Psychoanalyse als »armes Ding«, als Aschenputtel, dem es nicht gelingt, sich aus seinen Dienstbarkeiten zu befreien. Grundlage der optimistischen wie der skeptischen Auffassung von der Funktion des Ichs ist seine Mittelstellung zwischen anderen Instanzen: Als »Grenzwesen« spricht Freud das Ich an, um seine Vermittlungsversuche zugleich in den Kontext von Lüge und Verstellung zu stellen: »liebedienerisch, opportunistisch und lügnerisch« ist das Ich, da es sich jederzeit strategisch verhalten muss wie ein Politiker, der die unangenehme Wahrheit vor der öffentlichen Meinung verbergen muss. Als scheinbarer Herrscher über die Welt des Bewusstseins ist das Ich zugleich ein »unterwürfiger Knecht» des Ichs, dessen mächtigem Willen er unterliegt, indem er ihm Raum schafft. Aus dem Löwen ist ein Fuchs geworden, dem die Klugheit nach dem Vorbild Graciáns die Kunst der Verstellung gebietet: »Nie tut sie das, was sie vorgibt, sondern zielt nur, um zu täuschen. Mit Geschicklichkeit macht sie Luftstreiche; dann aber führt sie in der Wirklichkeit etwas Unerwartetes aus, stets darauf bedacht, ihr Spiel zu verbergen. $\aleph^{18}$ Zielt Graciáns Lob der List auf ein souveränes Ich, das durch die Odysseus abgelesene Kunst der Affektbeherrschung situativ richtig handelt, um den eigenen Anspruch durchzusetzen, so ist Freuds Ich jedoch ein betrogener Betrüger, ein Held, der beständig in die Stricke der eigenen Anschläge fällt. Als Grenzwesen muss das Ich der Verstellung vertrauen, um das eigene Überleben in einem Zwischenbereich zu sichern, den es nicht verlassen kann. So gehen Liminalität und dissimulatio in Freuds Theorie des Ichs eine Symbiose ein, die sich in ähnlicher Weise wie bei Nietzsche einer negativen Anthropologie verdankt und dem Lob des Grenzgängers eine andere, skeptische Weise abgewinnt.

18 Balthasar Gracián: Handorakel und Kunst der Weltklugheit, Stuttgart 1954, S. 10. 


\section{Liminale Psychoanalyse}

Wenn Freud die Traumdeutung als sein Jahrhundertbuch bezeichnet, so setzt er die Geburt der Psychoanalyse nicht nur symbolträchtig an die Schwelle des 20. Jahrhunderts. Er formuliert damit einen Herrschaftsanspruch, den die Psychoanalyse in den letzten Jahrzehnten verloren hat. Selbst die postmoderne Dezentrierung des Subjekts, die der Psychoanalyse viel zu verdanken schien, hat sich von Freud abgesetzt, um eigene, weiterführende Modelle vorzuschlagen. ${ }^{19}$ Während Michel Foucault sich schon 1954 für das gleichzeitige Erscheinen von Freuds Traumdeutung und Husserls Logischen Untersuchungen interessiert, um auf überraschende Weise die Partei der Phänomenologie zu ergreifen, legt Derrida in seinen Überlegungen zu den Szenen der Schrift bei Freud ein graphisches Modell des Unbewussten vor, das er in die eigene Theorie der différance als Spur (trace) und Bahnung (frayage) überführt. Wie viel Derrida auch immer Freuds Theorie der Erinnerungsspuren aus Jenseits des Lustprinzips verdanken mag: Der Überführung des psychischen Modells Freuds in eine Graphie des Unbewussten im Kontext der Literalität steht der liminale Aspekt der Psychoanalyse zur Seite, der sich sowohl in Freuds Interesse für Schwellenphänomene wie den Traum zeigt als auch in seiner eigenen Theorie des Ichs als Grenzwesen, das sich zwischen zwei fremden und bedrohlichen Welten behaupten muss. Von Freuds Schrift, die selbst immer wieder die Grenze von Wissenschaft und Literatur überschreitet, einen Aufschluss über den Zusammenhang von Literalität und Liminalität zu erwarten, kann daher nicht heißen, der Psychoanalyse einen eindeutig fixierbaren Ort in der Geschichte des 20. Jahrhunderts zu geben, wie es die Kritik immer wieder getan hat. Freud nach 150 Jahren noch ernst zu nehmen bedeutet vielmehr, wie schon Samuel Weber es nahegelegt hat, den Strategien der »Verzerrung, Verschiebung und Verstellung ${ }^{20}{ }^{2}$ nachzugehen, die Freuds Schriften selbst in einen Raum des Zwischen versetzen, den zu verlassen bedeutet, die Psychoanalyse auf ihrem Weg in die Unterwelt zu verfehlen. Den Abgrund bewegen, wenn die's droben auch weigern, vermag die Psychoanalyse als eine Schwellenkunde in einer Zeit, die nach Walter Benjamin an Schwellenerfahrungen arm geworden ist. Wenn das Einschlafen vielleicht die einzige ist, die uns geblieben ist, dann ist der Psychoanalyse ein gutes Erwachen im 21. Jahrhundert zu wünschen.

19 Vgl. Achim Geisenhanslüke: »Freud aus Frankreich. Psychoanalyse und Postmoderne«, in: Kodikas/Code 23 (2000), S. 274-285.

20 Samuel Weber: Freud-Legende. Vier Studien zum psychoanalytischen Denken, Wien 2002, S. 40. 


\section{Literatur}

Anzieu, Didier: Freuds Selbstanalyse und die Entdeckung der Psychoanalyse, Stuttgart 1990.

Benjamin, Walter: Das Passagen-Werk. Erster Band, Frankfurt a.M. 1982.

Braungart, Wolfgang/Fischer-Lichte, Erika: Ritual und Literatur, Tübingen 1996.

Buck-Morss, Susan: Dialektik des Sehens. Walter Benjamin und das Passagen-Werk, Frankfurt a.M. 1993.

Derrida, Jacques: Schibboleth. Für Paul Celan, Wien 1986.

Freud, Sigmund: Gesammelte Werke. Chronologisch geordnet. Unter Mitwirkung von Marie Bonaparte, Prinzessin Georg von Griechenland, herausgegeben von Anna Freud, Frankfurt a.M. 1999.

Gennep, Arnold van: Übergangsriten (Les rites de passage), Frankfurt a.M., New York 1999.

Geisenhanslüke, Achim: »Freud aus Frankreich. Psychoanalyse und Postmoderne«, in: Kodikas/Code 23 (2000), S. 274-285.

Gracián, Balthasar: Handorakel und Kunst der Weltklugheit, Stuttgart 1954.

Hohnsträter, Dirk: »Im Zwischenraum. Ein Lob des Grenzgängers«, in: Claudia Benthien/Irmela Marei Krüger-Fürhoff (Hg.), Über Grenzen. Limitation und Transgression in Literatur und Ästhetik, Stuttgart, Weimar 1999, S. 231-244.

Illouz, Eva: Der Konsum der Romantik. Liebe und die kulturellen Widersprüche des Kapitalismus, Frankfurt a.M., New York 2003.

Menninghaus, Wolfgang: Schwellenkunde. Walter Benjamins Passage des Mythos, Frankfurt a.M. 1986.

Pikulik, Lothar: »Schwelle und Übergang. Zu einem Schlüsselmotiv der Romantik«, in: Aurora 53 (1993), S. 13-24.

Platthaus, Isabel: Höllenfahrten. Die epische katábasis und die Unterwelten der Moderne, München 2004.

Saul, Nicholas/Steuer, Daniel/Möbius, Frank/Illner, Birgit: Schwellen. Germanistische Erkundungen einer Metapher, Würzburg 1999.

Starobinski, Jean/Grubrich-Simitis, Ilse/Solms, Mark: Hundert Jahre >Traumdeutung< von Sigmund Freud. Drei Essays, Frankfurt a.M. 2000.

Turner, Victor: Das Ritual. Struktur und Anti-Struktur, Frankfurt a.M., New York 2005.

Weber, Samuel: Freud-Legende. Vier Studien zum psychoanalytischen Denken, Wien 2002. 